Acta Crystallographica Section E

Structure Reports

Online

ISSN 1600-5368

\section{3-Benzyl-2,4,6-triphenylpyrylium tetrafluoroborate}

\section{Francisco Jara, ${ }^{\mathrm{a}}$ Moisés Domínguez, ${ }^{a}$ Marcos C. Rezende, ${ }^{a}$ Edward R. T. Tiekink, ${ }^{b} *$ James L. Wardell ${ }^{\mathrm{c}}$ and Solange M. S. V. Wardell ${ }^{d} \ddagger$}

${ }^{\text {a }}$ Facultad de Quimica y Biologia, Universidad de Santiago, Casilla 40, Correo 33, Santiago, Chile, ${ }^{\mathbf{b}}$ Department of Chemistry, The University of Texas at San Antonio, 6900 North Loop 1604 West, San Antonio, Texas 78249-0698, USA, ${ }^{\mathrm{c}}$ Department of Chemistry, University of Aberdeen, Old Aberdeen AB24 3UE, Scotland, and, Instituto de Química, Universidade Federal do Rio de Janeiro, 21945-970 Rio de Janeiro, RJ, Brazil, and d Complexo Tecnológico de Medicamentos Farmanguinhos, Av. Comandante Guaranys 447, Jacarepaguá - Rio de Janeiro, RJ, Brazil

₹ Additional correspondence e-mail: solangewardell@yahoo.co.uk

Correspondence e-mail: edward.tiekink@utsa.edu

\section{Key indicators}

Single-crystal X-ray study

$T=120 \mathrm{~K}$

Mean $\sigma(\mathrm{C}-\mathrm{C})=0.003 \AA$

$R$ factor $=0.055$

$w R$ factor $=0.142$

Data-to-parameter ratio $=12.7$

For details of how these key indicators were automatically derived from the article, see http://journals.iucr.org/e.
(C) 2006 International Union of Crystallography All rights reserved
The central $\mathrm{OC}_{5}$ ring in the cation of the title compound, $\mathrm{C}_{30} \mathrm{H}_{23} \mathrm{O}^{+} \cdot \mathrm{BF}_{4}{ }^{-}$, has considerable aromatic character but the pendant aromatic rings are not coplanar. The crystal structure comprises undulating layers of cations separated by $\mathrm{BF}_{4}^{-}$ anions with significant intermolecular interactions between them.

\section{Comment}

The structure of the title compound, (I) (Fig. 1 and Table 1), shows significant twisting of the pendant aromatic rings out of the central plane. The dihedral angles between the O1/C1-C5 ring and the $\mathrm{C} 6-\mathrm{C} 11, \mathrm{C} 12-\mathrm{C} 17$ and $\mathrm{C} 25-\mathrm{C} 30$ rings are $28.14(10), 56.70(11)$ and $83.44(10)^{\circ}$, respectively. Within the central ring, which formally carries a positive charge, the two $\mathrm{O}-\mathrm{C}$ distances are equal within experimental error and the $\mathrm{C}-\mathrm{C}$ distances lie in the relatively narrow range 1.361 (3)1.414 (3) §. These observations strongly suggest substantial delocalization of $\pi$-electron density over this ring.

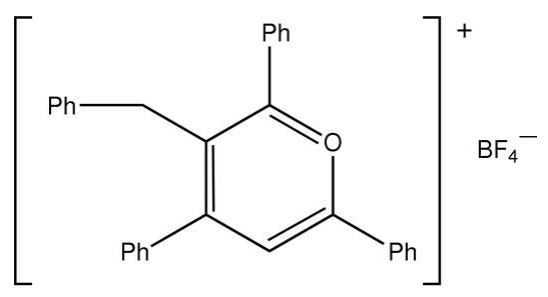

(I)

In the crystal structure there are a number of intermolecular interactions linking the ions. The primary interactions operating in the crystal structure are illustrated in Fig. 2. Here, $B-F \cdots H$ contacts are highlighted as golden dashed lines. The first contact occurs between the two components of the asymmetric unit so that $\mathrm{C} 20-\mathrm{H} 20 \cdots \mathrm{F} 4$ is $2.49 \AA, \mathrm{C} 20 \cdots \mathrm{F} 4$ is 3.355 (3) $\AA$ and the angle at $\mathrm{H} 20$ is $152^{\circ}$. The second $\mathrm{F} \cdot \mathrm{H}$ contact involves the $\mathrm{C} 8^{\mathrm{i}}$ and $\mathrm{F} 3$ atoms so that $\mathrm{C} 8{ }^{\mathrm{i}}-\mathrm{H} 8^{\mathrm{i}} \cdots \mathrm{F} 3$ is $2.55 \AA, \mathrm{C} 8{ }^{\mathrm{i}} \cdot \mathrm{F} 3$ is 3.321 (3) $\AA$ and the angle at $\mathrm{H} 8$ is $139 \AA$ [symmetry code: (i) $1-x, 1-y,-z$ ]. The remaining two $\mathrm{F}$ atoms serve to link two central O1/C1-C5 rings. The parameters associated with these interactions are $\mathrm{B} 1-\mathrm{F} 1 \cdots$ ring centroid $(\mathrm{O} 1 / \mathrm{C} 1-\mathrm{C} 5)=3.091$ (2) $\AA$ and angle at $\mathrm{F} 1=114.52(15)^{\circ}$, and $\mathrm{B} 1-\mathrm{F} 2 \cdots \operatorname{ring}$ centroid $(\mathrm{O} 1 / \mathrm{C} 1-\mathrm{C} 5)^{\mathrm{ii}}=$ 3.080 (2) $\AA$ and angle at $\mathrm{F} 2=118.59(17)^{\circ}$ [symmetry code: (ii) $\left.1-x, \frac{1}{2}+y, \frac{1}{2}-z\right]$. Formally, these might be considered as $\mathrm{F}$. - ring centroid(O1/C1-C5) interactions, but as seen in the $\mathrm{B}-\mathrm{F} \cdots$ ring centroid(O1/C1-C5) angles there is significant bending in the angles so that $\mathrm{F} 1$ approaches atoms $\mathrm{O} 1$ and $\mathrm{C} 1$ at 2.9508 (24) and 2.9766 (28) $\AA$, respectively, with the other F1 …C distances being greater than $3.3 \AA$. A similar situation pertains for the interactions involving atom F2. Thus, the
Received 12 July 2006 Accepted 20 July 2006 


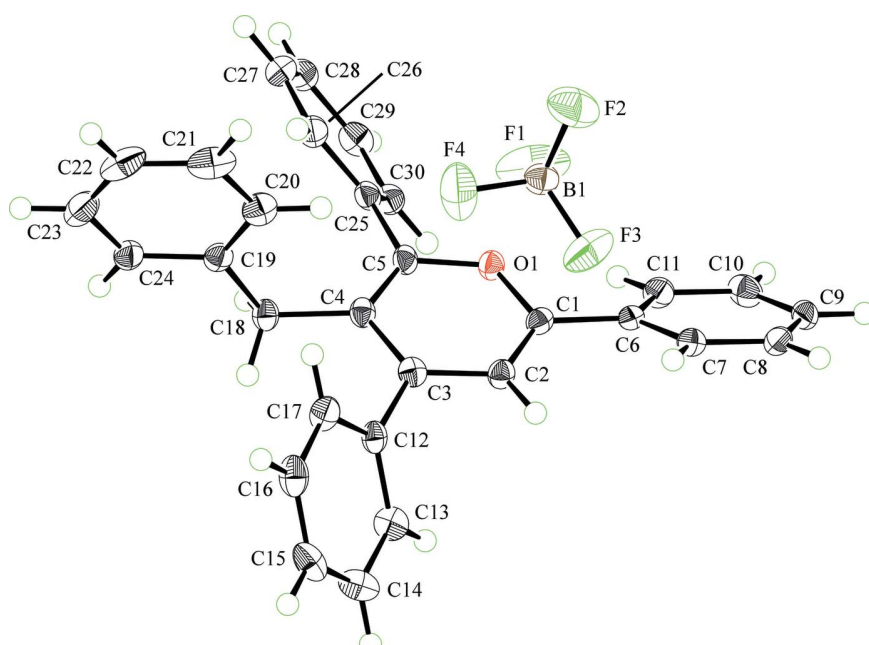

Figure 1

The asymmetric unit of (I) showing $50 \%$ probability displacement ellipsoids and the atom-numbering scheme.

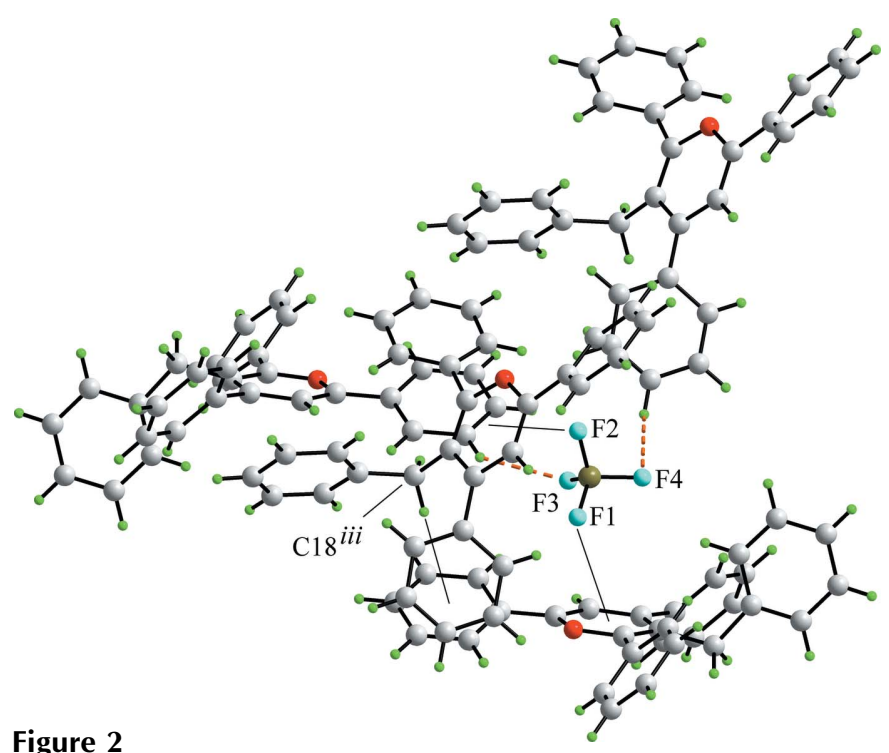

Environment about the $\mathrm{BF}_{4}{ }^{-}$anion in (I) (Crystal Impact, 2006). Color code: O (red), C (grey), B (brown) and H (green).

$\mathrm{F} 2 \cdots \mathrm{O} 1^{\mathrm{ii}}, \mathrm{F} 2 \cdots \mathrm{C} 1^{\mathrm{ii}}$ and $\mathrm{F} 2 \cdots \mathrm{C} 5^{\mathrm{ii}}$ distances are 2.9270 (22), 3.1280 (28) and 3.1845 (26) $\AA$, respectively, the remaining F2 . . C $\mathrm{C}^{\mathrm{ii}}$ distances being greater than $3.5 \AA$. The only other significant intermolecular contact in the structure of (I) is also illustrated in Fig. 2, i.e. a $\mathrm{C} 18^{\mathrm{iii}}-\mathrm{H} 18 A^{\mathrm{iii}}$. . ring centroid(C6C11) contact with an $\mathrm{H} 18 A^{\mathrm{iii}}$..ring centroid distance of $2.98 \AA$ and an angle of $152^{\circ}$ at the $\mathrm{H} 18 A^{\mathrm{iii}}$ atom. The global crystal structure may be described as comprising undulating layers of cations interspersed with anions (see Fig. 3).

\section{Experimental}

The title compound was isolated as a by-product in the preparation of 2-(4-hyroxyphenyl)-4,6-diphenylpyrylium tetrafluoroborate from 1,3diphenylpropenone and 4-hydroxyphenylethanone in the presence of boron trifluoride etherate (Aliaga et al., 1997). The two products were separated by fractional crystallization from acetic acid. The title compound, recrystallized from $\mathrm{AcOH}$, had melting point and spec-

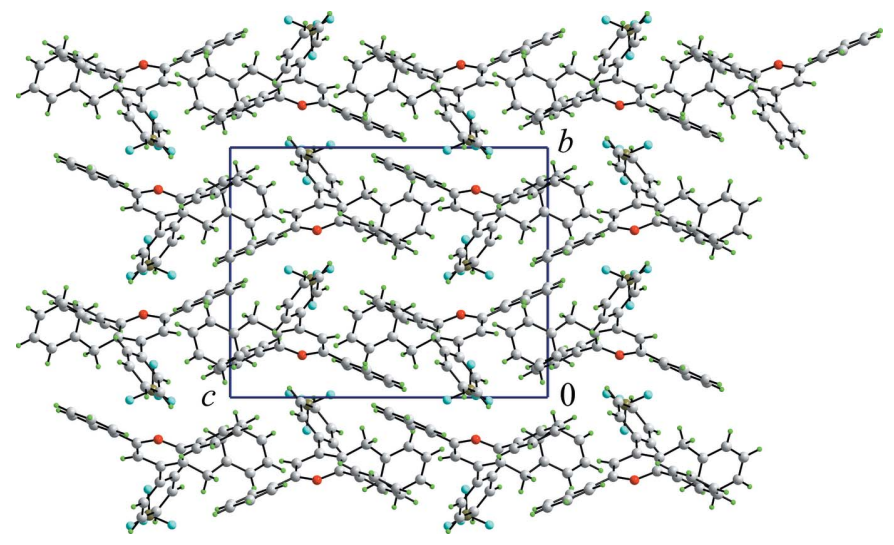

Figure 3

Packing diagram for (I), viewed approximately down the $a$ axis (Crystal Impact, 2006). Color code as for Fig. 2.

troscopic properties in agreement with literature values (Marton et al., 1999).

\section{Crystal data}

$\mathrm{C}_{30} \mathrm{H}_{23} \mathrm{O}^{+} \cdot \mathrm{BF}^{4-}$

$M_{r}=486.29$

Monoclinic, $P 2_{1} / c$

$a=10.6170(3) \AA$

$b=13.2065(3) \AA$

$c=17.1985(5) \AA$

$\beta=102.784(1)^{\circ}$

$V=2351.68(11) \AA^{3}$

$Z=4$

$D_{x}=1.374 \mathrm{Mg} \mathrm{m}^{-3}$

Mo $K \alpha$ radiation

$\mu=0.10 \mathrm{~mm}^{-1}$

$T=120$ (2) $\mathrm{K}$

Block, pale yellow

$0.24 \times 0.10 \times 0.08 \mathrm{~mm}$

\section{Data collection}

Bruker-Nonius KappaCCD diffractometer

$\varphi$ and $\omega$ scans

Absorption correction: multi-scan (SADABS; Sheldrick, 2003)

$T_{\min }=0.834, T_{\max }=1$

\section{Refinement}

Refinement on $F^{2}$

$R\left[F^{2}>2 \sigma\left(F^{2}\right)\right]=0.055$

$w R\left(F^{2}\right)=0.142$

$S=1.05$

4136 reflections

325 parameters

$\mathrm{H}$-atom parameters constrained

$$
\begin{aligned}
& 25644 \text { measured reflections } \\
& 4136 \text { independent reflections } \\
& 3347 \text { reflections with } I>2 \sigma(I) \\
& R_{\text {int }}=0.042 \\
& \theta_{\max }=25.0^{\circ}
\end{aligned}
$$

Table 1

Selected geometric parameters $\left(\AA,{ }^{\circ}\right)$.

\begin{tabular}{lrrr}
\hline $\mathrm{O} 1-\mathrm{C} 1$ & $1.341(3)$ & $\mathrm{C} 2-\mathrm{C} 3$ & $1.397(3)$ \\
$\mathrm{O} 1-\mathrm{C} 5$ & $1.350(3)$ & $\mathrm{C} 3-\mathrm{C} 4$ & $1.414(3)$ \\
$\mathrm{C} 1-\mathrm{C} 2$ & $1.361(3)$ & $\mathrm{C} 4-\mathrm{C} 5$ & $1.381(3)$ \\
& & & \\
$\mathrm{C} 1-\mathrm{O} 1-\mathrm{C} 5$ & $122.38(17)$ & & \\
\hline
\end{tabular}

All $\mathrm{H}$ atoms were allowed to ride on their parent atoms in the riding-model approximation at $\mathrm{C}-\mathrm{H}$ distances of $0.95-0.99 \AA$, and with $U_{\text {iso }}(\mathrm{H})=1.2 U_{\text {eq }}(\mathrm{C})$. Evidence of some disorder in the position of the $\mathrm{BF}_{4}{ }^{-}$anion can be noted from Fig. 1. However, multiple positions could not be resolved.

Data collection: COLLECT (Hooft, 1998); cell refinement: DENZO (Otwinowski \& Minor, 1997) and COLLECT; data reduc- 
tion: DENZO and COLLECT; program(s) used to solve structure: SIR92 (Altomare et al., 1994); program(s) used to refine structure: SHELXL97 (Sheldrick, 1997); molecular graphics: ORTEPII (Johnson, 1976) and DIAMOND (Crystal Impact, 2006); software used to prepare material for publication: SHELXL97.

The authors thank the EPSRC X-ray Crystallographic Service, University of Southampton, for the data collection.

\section{References}

Aliaga, C., Galdames, J. S. \& Rezende, M. C. (1997). J. Chem. Soc. Perkin Trans 2, pp. 1055-1058.
Altomare, A., Cascarano, M., Giacovazzo, C., Guagliardi, A., Burla, M. C., Polidori, G. \& Camalli, M. (1994). J. Appl. Cryst. 27, 435.

Crystal Impact (2006). DIAMOND. Version 3.1. Crystal Impact GbR, Bonn, Germany.

Hooft, R. W. W. (1998). COLLECT. Nonius BV, Delft, The Netherlands.

Johnson, C. K. (1976). ORTEPII. Report ORNL-5138. Oak Ridge National Laboratory, Tennessee, USA.

Marton, A. L., Marton, G. I., Feaghici, C. \& Balaban, A. T. (1999). Rev. Roum. Chim. 44, 677-682.

Otwinowski, Z. \& Minor, W. (1997). Methods in Enzymology, Vol. 276, Macromolecular Crystallography, Part A, edited by C. W. Carter Jr \& R. M. Sweet, pp. 307-326. New York: Academic Press.

Sheldrick, G. M. (1997). SHELXL97. University of Göttingen, Germany. Sheldrick, G. M. (2003). SADABS. Version 2.10. Bruker AXS Inc., Madison, Wisconsin, USA. 


\section{supporting information}

Acta Cryst. (2006). E62, o3495-o3497 [https://doi.org/10.1107/S1600536806028285]

\section{3-Benzyl-2,4,6-triphenylpyrylium tetrafluoroborate}

Francisco Jara, Moisés Domínguez, Marcos C. Rezende, Edward R. T. Tiekink, James L. Wardell and Solange M. S. V. Wardell

3-Benzyl-2,4,6-triphenylpyrylium tetrafluoroborate

Crystal data

$\mathrm{C}_{30} \mathrm{H}_{23} \mathrm{O}^{+} \cdot \mathrm{BF}_{4}^{-}$

$M_{r}=486.29$

Monoclinic, $P 2_{1} / c$

Hall symbol: -P $2 \mathrm{ybc}$

$a=10.6170(3) \AA$

$b=13.2065(3) \AA$

$c=17.1985(5) \AA$

$\beta=102.784(1)^{\circ}$

$V=2351.68(11) \AA^{3}$

$Z=4$

$F(000)=1008$

$D_{\mathrm{x}}=1.374 \mathrm{Mg} \mathrm{m}^{-3}$

Mo $K \alpha$ radiation, $\lambda=0.71069 \AA$

Cell parameters from 5538 reflections

$\theta=2.9-27.5^{\circ}$

$\mu=0.10 \mathrm{~mm}^{-1}$

$T=120 \mathrm{~K}$

Block, pale yellow

$0.24 \times 0.10 \times 0.08 \mathrm{~mm}$

\section{Data collection}

Bruker-Nonius 95mm CCD camera on $\kappa$ goniostat diffractometer

Radiation source: Bruker-Nonius FR591 rotating anode

Graphite monochromator

Detector resolution: 9.091 pixels $\mathrm{mm}^{-1}$

$\varphi$ and $\omega$ scans

Absorption correction: multi scan

(SADABS; Sheldrick, 2003)

Refinement

Refinement on $F^{2}$

Least-squares matrix: full

$R\left[F^{2}>2 \sigma\left(F^{2}\right)\right]=0.055$

$w R\left(F^{2}\right)=0.142$

$S=1.05$

4136 reflections

325 parameters

0 restraints

Primary atom site location: structure-invariant direct methods

$T_{\min }=0.834, T_{\max }=1$

25644 measured reflections

4136 independent reflections

3347 reflections with $I>2 \sigma(I)$

$R_{\text {int }}=0.042$

$\theta_{\max }=25.0^{\circ}, \theta_{\min }=3.0^{\circ}$

$h=-12 \rightarrow 12$

$k=-15 \rightarrow 15$

$l=-20 \rightarrow 20$

Secondary atom site location: difference Fourier map

Hydrogen site location: inferred from neighbouring sites

$\mathrm{H}$-atom parameters constrained

$w=1 /\left[\sigma^{2}\left(F_{\mathrm{o}}^{2}\right)+(0.0667 P)^{2}+2.1496 P\right]$

where $P=\left(F_{\mathrm{o}}^{2}+2 F_{\mathrm{c}}^{2}\right) / 3$

$(\Delta / \sigma)_{\max }<0.001$

$\Delta \rho_{\max }=0.80$ e $\AA^{-3}$

$\Delta \rho_{\min }=-0.40$ e $\AA^{-3}$ 


\section{Special details}

Geometry. All e.s.d.'s (except the e.s.d. in the dihedral angle between two 1.s. planes) are estimated using the full covariance matrix. The cell e.s.d.'s are taken into account individually in the estimation of e.s.d.'s in distances, angles and torsion angles; correlations between e.s.d.'s in cell parameters are only used when they are defined by crystal symmetry. An approximate (isotropic) treatment of cell e.s.d.'s is used for estimating e.s.d.'s involving l.s. planes.

Refinement. Refinement of $F^{2}$ against ALL reflections. The weighted $R$-factor $w R$ and goodness of fit $S$ are based on $F^{2}$, conventional $R$-factors $R$ are based on $F$, with $F$ set to zero for negative $F^{2}$. The threshold expression of $F^{2}>\sigma\left(F^{2}\right)$ is used only for calculating $R$-factors (gt) etc. and is not relevant to the choice of reflections for refinement. $R$-factors based on $F^{2}$ are statistically about twice as large as those based on $F$, and $R$ - factors based on ALL data will be even larger.

Fractional atomic coordinates and isotropic or equivalent isotropic displacement parameters $\left(\AA^{2}\right)$

\begin{tabular}{|c|c|c|c|c|}
\hline & $x$ & $y$ & $z$ & $U_{\text {iso }} * / U_{\text {eq }}$ \\
\hline F1 & $0.57576(17)$ & $0.50732(15)$ & $0.27361(13)$ & $0.0707(7)$ \\
\hline $\mathrm{F} 2$ & $0.71621(18)$ & $0.63183(12)$ & $0.25861(12)$ & $0.0559(5)$ \\
\hline F3 & $0.70332(18)$ & $0.48944(15)$ & $0.18351(10)$ & $0.0588(5)$ \\
\hline $\mathrm{F} 4$ & $0.78734(18)$ & $0.48523(15)$ & $0.31391(11)$ & $0.0636(5)$ \\
\hline $\mathrm{O} 1$ & $0.40466(14)$ & $0.33118(11)$ & $0.27331(9)$ & $0.0202(3)$ \\
\hline $\mathrm{C} 1$ & $0.4465(2)$ & $0.31678(16)$ & $0.20605(13)$ & $0.0190(5)$ \\
\hline $\mathrm{C} 2$ & $0.5580(2)$ & $0.26442(16)$ & $0.20922(13)$ & $0.0201(5)$ \\
\hline $\mathrm{H} 2$ & 0.5889 & 0.2550 & 0.1620 & $0.024 *$ \\
\hline $\mathrm{C} 3$ & $0.6273(2)$ & $0.22436(16)$ & $0.28119(13)$ & $0.0200(5)$ \\
\hline $\mathrm{C} 4$ & $0.5820(2)$ & $0.24083(16)$ & $0.35153(13)$ & $0.0193(5)$ \\
\hline $\mathrm{C} 5$ & $0.4691(2)$ & $0.29537(16)$ & $0.34461(13)$ & $0.0195(5)$ \\
\hline C6 & $0.3624(2)$ & $0.36022(16)$ & $0.13507(13)$ & $0.0187(5)$ \\
\hline $\mathrm{C} 7$ & $0.4156(2)$ & $0.38924(16)$ & $0.07108(13)$ & $0.0216(5)$ \\
\hline $\mathrm{H} 7$ & 0.5057 & 0.3818 & 0.0744 & $0.026^{*}$ \\
\hline $\mathrm{C} 8$ & $0.3370(2)$ & $0.42882(17)$ & $0.00296(13)$ & $0.0245(5)$ \\
\hline H8 & 0.3727 & 0.4482 & -0.0408 & $0.029 *$ \\
\hline C9 & $0.2061(2)$ & $0.44013(17)$ & $-0.00133(14)$ & $0.0260(5)$ \\
\hline H9 & 0.1523 & 0.4681 & -0.0479 & $0.031 *$ \\
\hline $\mathrm{C} 10$ & $0.1527(2)$ & $0.41098(18)$ & $0.06188(15)$ & $0.0281(6)$ \\
\hline H10 & 0.0627 & 0.4188 & 0.0584 & $0.034 *$ \\
\hline C11 & $0.2304(2)$ & $0.37063(17)$ & $0.12982(14)$ & $0.0242(5)$ \\
\hline H11 & 0.1938 & 0.3500 & 0.1729 & $0.029 *$ \\
\hline C12 & $0.7417(2)$ & 0.16057 (17) & $0.27859(13)$ & $0.0207(5)$ \\
\hline $\mathrm{C} 13$ & $0.7277(2)$ & $0.07825(18)$ & $0.22708(15)$ & $0.0268(5)$ \\
\hline H13 & 0.6450 & 0.0621 & 0.1954 & $0.032 *$ \\
\hline $\mathrm{C} 14$ & $0.8336(2)$ & $0.01974(19)$ & $0.22177(16)$ & $0.0324(6)$ \\
\hline H14 & 0.8229 & -0.0366 & 0.1866 & $0.039 *$ \\
\hline $\mathrm{C} 15$ & $0.9547(2)$ & $0.04247(18)$ & $0.26704(15)$ & $0.0305(6)$ \\
\hline H15 & 1.0270 & 0.0019 & 0.2633 & $0.037^{*}$ \\
\hline C16 & $0.9694(2)$ & 0.12461 (19) & $0.31768(15)$ & $0.0298(6)$ \\
\hline H16 & 1.0528 & 0.1412 & 0.3483 & $0.036^{*}$ \\
\hline $\mathrm{C} 17$ & $0.8643(2)$ & 0.18334 (19) & $0.32446(14)$ & $0.0271(5)$ \\
\hline H17 & 0.8755 & 0.2391 & 0.3603 & $0.033 *$ \\
\hline C18 & $0.6405(2)$ & 0.18787 (17) & $0.42883(13)$ & $0.0213(5)$ \\
\hline H18A & 0.6771 & 0.1228 & 0.4156 & $0.026^{*}$ \\
\hline
\end{tabular}




$\begin{array}{lllll}\text { H18B } & 0.5698 & 0.1715 & 0.4558 & 0.026^{*} \\ \text { C19 } & 0.7452(2) & 0.24305(17) & 0.48847(13) & 0.0213(5) \\ \text { C20 } & 0.7977(2) & 0.33477(18) & 0.47235(15) & 0.0278(5) \\ \text { H20 } & 0.7641 & 0.3688 & 0.4235 & 0.033^{*} \\ \text { C21 } & 0.8997(2) & 0.3768(2) & 0.52801(17) & 0.0366(6) \\ \text { H21 } & 0.9354 & 0.4395 & 0.5166 & 0.044^{*} \\ \text { C22 } & 0.9499(2) & 0.3289(2) & 0.59937(16) & 0.0376(7) \\ \text { H22 } & 1.0217 & 0.3571 & 0.6359 & 0.045^{*} \\ \text { C23 } & 0.8946(2) & 0.2396(2) & 0.61708(15) & 0.0344(6) \\ \text { H23 } & 0.9263 & 0.2073 & 0.6669 & 0.041^{*} \\ \text { C24 } & 0.7929(2) & 0.19681(18) & 0.56234(13) & 0.0249(5) \\ \text { H24 } & 0.7552 & 0.1355 & 0.5751 & 0.030^{*} \\ \text { C25 } & 0.3994(2) & 0.31854(16) & 0.40788(13) & 0.0207(5) \\ \text { C26 } & 0.4613(2) & 0.36439(17) & 0.47898(14) & 0.0247(5) \\ \text { H26 } & 0.5509 & 0.3796 & 0.4884 & 0.030^{*} \\ \text { C27 } & 0.3916(2) & 0.38770(18) & 0.53592(14) & 0.0284(5) \\ \text { H27 } & 0.4333 & 0.4201 & 0.5841 & 0.034^{*} \\ \text { C28 } & 0.2617(2) & 0.36415(18) & 0.52307(14) & 0.0277(6) \\ \text { H28 } & 0.2147 & 0.3797 & 0.5626 & 0.033^{*} \\ \text { C29 } & 0.2000(2) & 0.31797(18) & 0.45261(15) & 0.0278(5) \\ \text { H29 } & 0.1109 & 0.3017 & 0.4440 & 0.033^{*} \\ \text { C30 } & 0.2680(2) & 0.29533(17) & 0.39466(14) & 0.0239(5) \\ \text { H30 } & 0.2253 & 0.2642 & 0.3462 & 0.029^{*} \\ \text { B1 } & 0.6920(3) & 0.5292(2) & 0.25495(17) & 0.0271(6)\end{array}$

Atomic displacement parameters $\left(\AA^{2}\right)$

\begin{tabular}{lllllll}
\hline & $U^{11}$ & $U^{22}$ & $U^{33}$ & $U^{12}$ & $U^{13}$ & $U^{23}$ \\
\hline F1 & $0.0457(10)$ & $0.0742(13)$ & $0.1045(17)$ & $-0.0265(9)$ & $0.0432(11)$ & $-0.0448(12)$ \\
F2 & $0.0666(12)$ & $0.0319(9)$ & $0.0727(13)$ & $-0.0064(8)$ & $0.0231(10)$ & $-0.0016(8)$ \\
F3 & $0.0723(12)$ & $0.0718(13)$ & $0.0360(10)$ & $-0.0067(10)$ & $0.0199(9)$ & $-0.0175(9)$ \\
F4 & $0.0644(12)$ & $0.0754(13)$ & $0.0489(11)$ & $0.0126(10)$ & $0.0083(9)$ & $0.0235(10)$ \\
O1 & $0.0210(8)$ & $0.0222(8)$ & $0.0177(8)$ & $0.0011(6)$ & $0.0051(6)$ & $0.0023(6)$ \\
C1 & $0.0221(11)$ & $0.0188(11)$ & $0.0171(11)$ & $-0.0036(9)$ & $0.0061(9)$ & $-0.0006(9)$ \\
C2 & $0.0204(11)$ & $0.0231(11)$ & $0.0180(11)$ & $0.0003(9)$ & $0.0065(9)$ & $0.0015(9)$ \\
C3 & $0.0183(11)$ & $0.0196(11)$ & $0.0224(12)$ & $-0.0035(9)$ & $0.0048(9)$ & $0.0017(9)$ \\
C4 & $0.0196(11)$ & $0.0191(11)$ & $0.0186(11)$ & $-0.0028(9)$ & $0.0030(9)$ & $0.0028(9)$ \\
C5 & $0.0229(11)$ & $0.0174(11)$ & $0.0178(11)$ & $-0.0026(9)$ & $0.0035(9)$ & $0.0038(9)$ \\
C6 & $0.0210(11)$ & $0.0173(11)$ & $0.0168(11)$ & $0.0012(9)$ & $0.0021(9)$ & $-0.0022(9)$ \\
C7 & $0.0223(11)$ & $0.0205(11)$ & $0.0217(12)$ & $0.0008(9)$ & $0.0042(10)$ & $-0.0008(9)$ \\
C8 & $0.0332(13)$ & $0.0218(12)$ & $0.0180(12)$ & $-0.0020(10)$ & $0.0046(10)$ & $-0.0011(9)$ \\
C9 & $0.0310(13)$ & $0.0194(11)$ & $0.0217(12)$ & $0.0014(10)$ & $-0.0071(10)$ & $-0.0012(9)$ \\
C10 & $0.0199(11)$ & $0.0271(13)$ & $0.0349(14)$ & $0.0039(10)$ & $0.0012(11)$ & $-0.0013(11)$ \\
C11 & $0.0217(11)$ & $0.0269(12)$ & $0.0242(13)$ & $0.0025(10)$ & $0.0055(10)$ & $-0.0008(10)$ \\
C12 & $0.0207(11)$ & $0.0239(12)$ & $0.0186(11)$ & $-0.0003(9)$ & $0.0066(9)$ & $0.0058(9)$ \\
C13 & $0.0216(11)$ & $0.0277(13)$ & $0.0314(13)$ & $-0.0040(10)$ & $0.0064(10)$ & $-0.0005(10)$ \\
C14 & $0.0325(14)$ & $0.0236(12)$ & $0.0440(16)$ & $0.0005(10)$ & $0.0150(12)$ & $-0.0025(11)$ \\
C15 & $0.0262(13)$ & $0.0280(13)$ & $0.0415(15)$ & $0.0067(10)$ & $0.0167(11)$ & $0.0132(11)$
\end{tabular}


supporting information

\begin{tabular}{lllllll}
\hline & & & & \\
C16 & $0.0207(12)$ & $0.0403(15)$ & $0.0281(13)$ & $0.0013(11)$ & $0.0046(10)$ & $0.0096(11)$ \\
C17 & $0.0250(12)$ & $0.0340(13)$ & $0.0220(12)$ & $0.0013(10)$ & $0.0044(10)$ & $0.0009(10)$ \\
C18 & $0.0211(11)$ & $0.0238(11)$ & $0.0195(12)$ & $-0.0002(9)$ & $0.0055(9)$ & $0.0038(9)$ \\
C19 & $0.0197(11)$ & $0.0253(12)$ & $0.0200(12)$ & $0.0033(9)$ & $0.0066(9)$ & $-0.0013(9)$ \\
C20 & $0.0268(12)$ & $0.0302(13)$ & $0.0285(13)$ & $-0.0028(10)$ & $0.0104(11)$ & $-0.0017(11)$ \\
C21 & $0.0319(14)$ & $0.0358(15)$ & $0.0476(17)$ & $-0.0100(11)$ & $0.0205(13)$ & $-0.0147(13)$ \\
C22 & $0.0247(13)$ & $0.0554(18)$ & $0.0333(15)$ & $-0.0026(12)$ & $0.0080(11)$ & $-0.0216(13)$ \\
C23 & $0.0291(13)$ & $0.0493(16)$ & $0.0233(13)$ & $0.0099(12)$ & $0.0024(11)$ & $-0.0080(12)$ \\
C24 & $0.0264(12)$ & $0.0281(12)$ & $0.0207(12)$ & $0.0063(10)$ & $0.0065(10)$ & $-0.0008(10)$ \\
C25 & $0.0241(11)$ & $0.0189(11)$ & $0.0204(12)$ & $0.0032(9)$ & $0.0077(9)$ & $0.0043(9)$ \\
C26 & $0.0250(12)$ & $0.0252(12)$ & $0.0239(13)$ & $0.0013(10)$ & $0.0052(10)$ & $0.0016(10)$ \\
C27 & $0.0354(14)$ & $0.0281(13)$ & $0.0214(12)$ & $0.0036(11)$ & $0.0055(11)$ & $-0.0022(10)$ \\
C28 & $0.0337(13)$ & $0.0281(13)$ & $0.0257(13)$ & $0.0072(10)$ & $0.0158(11)$ & $0.0035(10)$ \\
C29 & $0.0248(12)$ & $0.0268(13)$ & $0.0342(14)$ & $0.0019(10)$ & $0.0115(11)$ & $0.0035(11)$ \\
C30 & $0.0253(12)$ & $0.0234(12)$ & $0.0234(12)$ & $0.0012(9)$ & $0.0061(10)$ & $0.0009(10)$ \\
B1 & $0.0270(14)$ & $0.0263(14)$ & $0.0298(15)$ & $0.0001(11)$ & $0.0099(12)$ & $-0.0016(12)$ \\
& & & & & \\
\hline
\end{tabular}

Geometric parameters $\left(\AA,{ }^{\circ}\right)$

\begin{tabular}{|c|c|c|c|}
\hline $\mathrm{F} 1-\mathrm{B} 1$ & $1.373(3)$ & $\mathrm{C} 14-\mathrm{H} 14$ & 0.9500 \\
\hline $\mathrm{F} 2-\mathrm{B} 1$ & $1.378(3)$ & $\mathrm{C} 15-\mathrm{C} 16$ & $1.378(4)$ \\
\hline F3-B1 & $1.366(3)$ & C15-H15 & 0.9500 \\
\hline $\mathrm{F} 4-\mathrm{B} 1$ & $1.392(3)$ & $\mathrm{C} 16-\mathrm{C} 17$ & $1.385(3)$ \\
\hline $\mathrm{O} 1-\mathrm{C} 1$ & $1.341(3)$ & $\mathrm{C} 16-\mathrm{H} 16$ & 0.9500 \\
\hline $\mathrm{O} 1-\mathrm{C} 5$ & $1.350(3)$ & C17-H17 & 0.9500 \\
\hline $\mathrm{C} 1-\mathrm{C} 2$ & $1.361(3)$ & $\mathrm{C} 18-\mathrm{C} 19$ & $1.521(3)$ \\
\hline $\mathrm{C} 1-\mathrm{C} 6$ & $1.461(3)$ & $\mathrm{C} 18-\mathrm{H} 18 \mathrm{~A}$ & 0.9900 \\
\hline $\mathrm{C} 2-\mathrm{C} 3$ & $1.397(3)$ & $\mathrm{C} 18-\mathrm{H} 18 \mathrm{~B}$ & 0.9900 \\
\hline $\mathrm{C} 2-\mathrm{H} 2$ & 0.9500 & $\mathrm{C} 19-\mathrm{C} 24$ & $1.400(3)$ \\
\hline $\mathrm{C} 3-\mathrm{C} 4$ & $1.414(3)$ & $\mathrm{C} 19-\mathrm{C} 20$ & $1.386(3)$ \\
\hline $\mathrm{C} 3-\mathrm{C} 12$ & $1.488(3)$ & $\mathrm{C} 20-\mathrm{C} 21$ & $1.392(4)$ \\
\hline $\mathrm{C} 4-\mathrm{C} 5$ & $1.381(3)$ & $\mathrm{C} 20-\mathrm{H} 20$ & 0.9500 \\
\hline $\mathrm{C} 4-\mathrm{C} 18$ & $1.509(3)$ & $\mathrm{C} 21-\mathrm{C} 22$ & $1.378(4)$ \\
\hline $\mathrm{C} 5-\mathrm{C} 25$ & $1.476(3)$ & $\mathrm{C} 21-\mathrm{H} 21$ & 0.9500 \\
\hline $\mathrm{C} 6-\mathrm{C} 11$ & $1.391(3)$ & $\mathrm{C} 22-\mathrm{C} 23$ & $1.382(4)$ \\
\hline $\mathrm{C} 6-\mathrm{C} 7$ & $1.397(3)$ & $\mathrm{C} 22-\mathrm{H} 22$ & 0.9500 \\
\hline $\mathrm{C} 7-\mathrm{C} 8$ & $1.382(3)$ & $\mathrm{C} 23-\mathrm{C} 24$ & $1.386(3)$ \\
\hline $\mathrm{C} 7-\mathrm{H} 7$ & 0.9500 & $\mathrm{C} 23-\mathrm{H} 23$ & 0.9500 \\
\hline $\mathrm{C} 8-\mathrm{C} 9$ & $1.384(3)$ & $\mathrm{C} 24-\mathrm{H} 24$ & 0.9500 \\
\hline $\mathrm{C} 8-\mathrm{H} 8$ & 0.9500 & $\mathrm{C} 25-\mathrm{C} 30$ & $1.398(3)$ \\
\hline $\mathrm{C} 9-\mathrm{C} 10$ & $1.387(3)$ & $\mathrm{C} 25-\mathrm{C} 26$ & $1.392(3)$ \\
\hline $\mathrm{C} 9-\mathrm{H} 9$ & 0.9500 & $\mathrm{C} 26-\mathrm{C} 27$ & $1.387(3)$ \\
\hline $\mathrm{C} 10-\mathrm{C} 11$ & $1.380(3)$ & $\mathrm{C} 26-\mathrm{H} 26$ & 0.9500 \\
\hline $\mathrm{C} 10-\mathrm{H} 10$ & 0.9500 & $\mathrm{C} 27-\mathrm{C} 28$ & $1.383(3)$ \\
\hline C11-H11 & 0.9500 & $\mathrm{C} 27-\mathrm{H} 27$ & 0.9500 \\
\hline $\mathrm{C} 12-\mathrm{C} 13$ & $1.389(3)$ & $\mathrm{C} 28-\mathrm{C} 29$ & $1.385(3)$ \\
\hline $\mathrm{C} 12-\mathrm{C} 17$ & $1.397(3)$ & $\mathrm{C} 28-\mathrm{H} 28$ & 0.9500 \\
\hline $\mathrm{C} 13-\mathrm{C} 14$ & $1.384(3)$ & $\mathrm{C} 29-\mathrm{C} 30$ & $1.386(3)$ \\
\hline
\end{tabular}




\begin{tabular}{|c|c|c|c|}
\hline $\mathrm{C} 13-\mathrm{H} 13$ & 0.9500 & $\mathrm{C} 29-\mathrm{H} 29$ & 0.9500 \\
\hline $\mathrm{C} 14-\mathrm{C} 15$ & $1.381(4)$ & $\mathrm{C} 30-\mathrm{H} 30$ & 0.9500 \\
\hline $\mathrm{C} 1-\mathrm{O} 1-\mathrm{C} 5$ & $122.38(17)$ & $\mathrm{C} 16-\mathrm{C} 17-\mathrm{H} 17$ & 120.0 \\
\hline $\mathrm{O} 1-\mathrm{C} 1-\mathrm{C} 2$ & $119.2(2)$ & $\mathrm{C} 12-\mathrm{C} 17-\mathrm{H} 17$ & 120.0 \\
\hline $\mathrm{O} 1-\mathrm{C} 1-\mathrm{C} 6$ & $114.02(18)$ & $\mathrm{C} 4-\mathrm{C} 18-\mathrm{C} 19$ & $117.97(18)$ \\
\hline $\mathrm{C} 2-\mathrm{C} 1-\mathrm{C} 6$ & $126.7(2)$ & $\mathrm{C} 4-\mathrm{C} 18-\mathrm{H} 18 \mathrm{~A}$ & 107.8 \\
\hline $\mathrm{C} 1-\mathrm{C} 2-\mathrm{C} 3$ & $120.7(2)$ & $\mathrm{C} 19-\mathrm{C} 18-\mathrm{H} 18 \mathrm{~A}$ & 107.8 \\
\hline $\mathrm{C} 1-\mathrm{C} 2-\mathrm{H} 2$ & 119.6 & $\mathrm{C} 4-\mathrm{C} 18-\mathrm{H} 18 \mathrm{~B}$ & 107.8 \\
\hline $\mathrm{C} 3-\mathrm{C} 2-\mathrm{H} 2$ & 119.6 & $\mathrm{C} 19-\mathrm{C} 18-\mathrm{H} 18 \mathrm{~B}$ & 107.8 \\
\hline $\mathrm{C} 2-\mathrm{C} 3-\mathrm{C} 4$ & $119.20(19)$ & $\mathrm{H} 18 \mathrm{~A}-\mathrm{C} 18-\mathrm{H} 18 \mathrm{~B}$ & 107.2 \\
\hline $\mathrm{C} 2-\mathrm{C} 3-\mathrm{C} 12$ & $117.49(19)$ & $\mathrm{C} 24-\mathrm{C} 19-\mathrm{C} 20$ & $118.8(2)$ \\
\hline $\mathrm{C} 4-\mathrm{C} 3-\mathrm{C} 12$ & $123.20(19)$ & $\mathrm{C} 24-\mathrm{C} 19-\mathrm{C} 18$ & $117.8(2)$ \\
\hline $\mathrm{C} 5-\mathrm{C} 4-\mathrm{C} 3$ & $117.36(19)$ & $\mathrm{C} 20-\mathrm{C} 19-\mathrm{C} 18$ & $123.3(2)$ \\
\hline $\mathrm{C} 5-\mathrm{C} 4-\mathrm{C} 18$ & $119.88(19)$ & $\mathrm{C} 19-\mathrm{C} 20-\mathrm{C} 21$ & $119.8(2)$ \\
\hline $\mathrm{C} 3-\mathrm{C} 4-\mathrm{C} 18$ & $122.05(19)$ & $\mathrm{C} 19-\mathrm{C} 20-\mathrm{H} 20$ & 120.1 \\
\hline $\mathrm{O} 1-\mathrm{C} 5-\mathrm{C} 4$ & $121.08(19)$ & $\mathrm{C} 21-\mathrm{C} 20-\mathrm{H} 20$ & 120.1 \\
\hline $\mathrm{O} 1-\mathrm{C} 5-\mathrm{C} 25$ & $111.18(18)$ & $\mathrm{C} 22-\mathrm{C} 21-\mathrm{C} 20$ & $121.2(2)$ \\
\hline $\mathrm{C} 4-\mathrm{C} 5-\mathrm{C} 25$ & $127.68(19)$ & $\mathrm{C} 22-\mathrm{C} 21-\mathrm{H} 21$ & 119.4 \\
\hline $\mathrm{C} 11-\mathrm{C} 6-\mathrm{C} 7$ & $119.9(2)$ & $\mathrm{C} 20-\mathrm{C} 21-\mathrm{H} 21$ & 119.4 \\
\hline $\mathrm{C} 11-\mathrm{C} 6-\mathrm{C} 1$ & $120.9(2)$ & $\mathrm{C} 21-\mathrm{C} 22-\mathrm{C} 23$ & $119.2(2)$ \\
\hline $\mathrm{C} 7-\mathrm{C} 6-\mathrm{C} 1$ & $119.14(19)$ & $\mathrm{C} 21-\mathrm{C} 22-\mathrm{H} 22$ & 120.4 \\
\hline $\mathrm{C} 8-\mathrm{C} 7-\mathrm{C} 6$ & $119.8(2)$ & $\mathrm{C} 23-\mathrm{C} 22-\mathrm{H} 22$ & 120.4 \\
\hline $\mathrm{C} 8-\mathrm{C} 7-\mathrm{H} 7$ & 120.1 & $\mathrm{C} 22-\mathrm{C} 23-\mathrm{C} 24$ & $120.2(2)$ \\
\hline $\mathrm{C} 6-\mathrm{C} 7-\mathrm{H} 7$ & 120.1 & $\mathrm{C} 22-\mathrm{C} 23-\mathrm{H} 23$ & 119.9 \\
\hline $\mathrm{C} 9-\mathrm{C} 8-\mathrm{C} 7$ & $119.8(2)$ & $\mathrm{C} 24-\mathrm{C} 23-\mathrm{H} 23$ & 119.9 \\
\hline $\mathrm{C} 9-\mathrm{C} 8-\mathrm{H} 8$ & 120.1 & $\mathrm{C} 19-\mathrm{C} 24-\mathrm{C} 23$ & $120.6(2)$ \\
\hline $\mathrm{C} 7-\mathrm{C} 8-\mathrm{H} 8$ & 120.1 & $\mathrm{C} 19-\mathrm{C} 24-\mathrm{H} 24$ & 119.7 \\
\hline $\mathrm{C} 8-\mathrm{C} 9-\mathrm{C} 10$ & $120.6(2)$ & $\mathrm{C} 23-\mathrm{C} 24-\mathrm{H} 24$ & 119.7 \\
\hline $\mathrm{C} 8-\mathrm{C} 9-\mathrm{H} 9$ & 119.7 & $\mathrm{C} 30-\mathrm{C} 25-\mathrm{C} 26$ & $119.9(2)$ \\
\hline $\mathrm{C} 10-\mathrm{C} 9-\mathrm{H} 9$ & 119.7 & $\mathrm{C} 30-\mathrm{C} 25-\mathrm{C} 5$ & $118.7(2)$ \\
\hline $\mathrm{C} 11-\mathrm{C} 10-\mathrm{C} 9$ & $120.0(2)$ & $\mathrm{C} 26-\mathrm{C} 25-\mathrm{C} 5$ & $121.4(2)$ \\
\hline $\mathrm{C} 11-\mathrm{C} 10-\mathrm{H} 10$ & 120.0 & $\mathrm{C} 27-\mathrm{C} 26-\mathrm{C} 25$ & $119.7(2)$ \\
\hline $\mathrm{C} 9-\mathrm{C} 10-\mathrm{H} 10$ & 120.0 & $\mathrm{C} 27-\mathrm{C} 26-\mathrm{H} 26$ & 120.2 \\
\hline $\mathrm{C} 6-\mathrm{C} 11-\mathrm{C} 10$ & $119.9(2)$ & $\mathrm{C} 25-\mathrm{C} 26-\mathrm{H} 26$ & 120.2 \\
\hline $\mathrm{C} 6-\mathrm{C} 11-\mathrm{H} 11$ & 120.1 & $\mathrm{C} 26-\mathrm{C} 27-\mathrm{C} 28$ & $120.4(2)$ \\
\hline $\mathrm{C} 10-\mathrm{C} 11-\mathrm{H} 11$ & 120.1 & $\mathrm{C} 26-\mathrm{C} 27-\mathrm{H} 27$ & 119.8 \\
\hline $\mathrm{C} 13-\mathrm{C} 12-\mathrm{C} 17$ & $118.9(2)$ & $\mathrm{C} 28-\mathrm{C} 27-\mathrm{H} 27$ & 119.8 \\
\hline $\mathrm{C} 13-\mathrm{C} 12-\mathrm{C} 3$ & $119.3(2)$ & $\mathrm{C} 29-\mathrm{C} 28-\mathrm{C} 27$ & $120.0(2)$ \\
\hline $\mathrm{C} 17-\mathrm{C} 12-\mathrm{C} 3$ & $121.7(2)$ & $\mathrm{C} 29-\mathrm{C} 28-\mathrm{H} 28$ & 120.0 \\
\hline $\mathrm{C} 12-\mathrm{C} 13-\mathrm{C} 14$ & $120.3(2)$ & $\mathrm{C} 27-\mathrm{C} 28-\mathrm{H} 28$ & 120.0 \\
\hline $\mathrm{C} 12-\mathrm{C} 13-\mathrm{H} 13$ & 119.8 & $\mathrm{C} 28-\mathrm{C} 29-\mathrm{C} 30$ & $120.3(2)$ \\
\hline $\mathrm{C} 14-\mathrm{C} 13-\mathrm{H} 13$ & 119.8 & $\mathrm{C} 28-\mathrm{C} 29-\mathrm{H} 29$ & 119.9 \\
\hline $\mathrm{C} 15-\mathrm{C} 14-\mathrm{C} 13$ & $120.7(2)$ & $\mathrm{C} 30-\mathrm{C} 29-\mathrm{H} 29$ & 119.9 \\
\hline $\mathrm{C} 15-\mathrm{C} 14-\mathrm{H} 14$ & 119.7 & $\mathrm{C} 25-\mathrm{C} 30-\mathrm{C} 29$ & 119.7 (2) \\
\hline $\mathrm{C} 13-\mathrm{C} 14-\mathrm{H} 14$ & 119.7 & $\mathrm{C} 25-\mathrm{C} 30-\mathrm{H} 30$ & 120.1 \\
\hline $\mathrm{C} 14-\mathrm{C} 15-\mathrm{C} 16$ & $119.3(2)$ & $\mathrm{C} 29-\mathrm{C} 30-\mathrm{H} 30$ & 120.1 \\
\hline $\mathrm{C} 14-\mathrm{C} 15-\mathrm{H} 15$ & 120.4 & $\mathrm{~F} 1-\mathrm{B} 1-\mathrm{F} 3$ & $113.1(2)$ \\
\hline
\end{tabular}




$\begin{array}{llll}\mathrm{C} 16-\mathrm{C} 15-\mathrm{H} 15 & 120.4 & \mathrm{~F} 1-\mathrm{B} 1-\mathrm{F} 2 & 111.4(2) \\ \mathrm{C} 17-\mathrm{C} 16-\mathrm{C} 15 & 120.9(2) & \mathrm{F} 3-\mathrm{B} 1-\mathrm{F} 2 & 111.5(2) \\ \mathrm{C} 17-\mathrm{C} 16-\mathrm{H} 16 & 119.6 & \mathrm{~F} 1-\mathrm{B} 1-\mathrm{F} 4 & 106.8(2) \\ \mathrm{C} 15-\mathrm{C} 16-\mathrm{H} 16 & 119.6 & \mathrm{~F} 3-\mathrm{B} 1-\mathrm{F} 4 & 107.5(2) \\ \mathrm{C} 16-\mathrm{C} 17-\mathrm{C} 12 & 119.9(2) & \mathrm{F} 2-\mathrm{B} 1-\mathrm{F} 4 & 106.2(2)\end{array}$

